\title{
Réformes des études médicales : les enjeux pour les enseignants et la spécialité de médecine d'urgence
}

\author{
Medical Curriculum Reforms: Issues for Teachers and the Specialty of Emergency Medicine
}

\author{
T. Pelaccia \\ (C) SFMU et Lavoisier SAS 2019
}

Les réformes actuellement menées dans les études de médecine, qui concernent tous les cycles de la formation, se caractérisent par un point commun : valoriser et accompagner le développement des compétences des étudiants, longtemps négligées au profit des connaissances scientifiques. L'évolution peut en apparence sembler minime. Cette perception est liée à une confusion fréquente, chez les enseignants, entre « compétences » et « connaissances », entretenue par le fait que ces dernières sont souvent catégorisées dans des groupes dont la dénomination - par exemple, le « savoir-faire » ou les « connaissances d'action » - laisse penser que « c'est déjà ce que l'on faisait ! ». Le passage d'une approche centrée sur les connaissances à une approche centrée sur les compétences est pourtant un véritable bouleversement, qui va profondément remettre en cause les pratiques actuelles de formation et d'évaluation des étudiants en médecine.

\section{Savoir utiliser plutôt que savoir}

Afin d'en saisir les enjeux, il est nécessaire d'effectuer un bref détour conceptuel, en clarifiant le terme de « compétences ». Celui-ci a été défini par de nombreux auteurs, y compris dans le domaine des sciences de la santé [1-3]. Selon ces définitions, être compétent ne signifie pas avoir beaucoup de connaissances, mais être capable d'utiliser ses connaissances (et d'autres ressources) pour agir efficacement dans les situations professionnelles, grâce à un raisonnement approprié. L'approche par compétences ne remet donc pas en cause l'importance des connaissances pour devenir médecin. Elle

T. Pelaccia $(\bowtie)$

Service d'aide médicale urgente du Bas-Rhin, hôpitaux universitaires de Strasbourg,

70, rue de l'Engelbreit, F-67200 Strasbourg, France

e-mail : pelaccia@unistra.fr

Centre de formation et de recherche en pédagogie des sciences de la santé (CFRPS), faculté de médecine de Strasbourg, université de Strasbourg,

4, rue Kirschleger, F-67085 Strasbourg, France permet de conceptualiser une perception à la fois partagée par beaucoup d'enseignants et documentée dans la littérature scientifique : ce n'est pas la quantité de connaissances qui compte, mais la façon dont celles-ci sont organisées dans le cerveau pour faciliter leur utilisation au bon moment et au bon endroit [4].

L'enjeu principal associé aux réformes des études de médecine est donc d'aider l'étudiant, non plus à accumuler dans sa mémoire à long terme des masses de connaissances dissociées les unes des autres, mais à créer des réseaux très riches et structurés de connaissances - couramment dénommés « scripts » [5] — qui pourront être mobilisés afin d'agir efficacement dans les situations professionnelles rencontrées par les futurs médecins.

\section{Former autrement}

Cette (r)évolution devrait s'accompagner d'une modification radicale du rapport des enseignants en médecine aux connaissances scientifiques. Dès lors que celles-ci ont pour but d'aider le futur médecin à agir efficacement en situation, leur apprentissage doit en effet être contextualisé. Pour comprendre ce que cela signifie, il est utile de rappeler qu'il existe, à travers le monde, deux grandes façons d'organiser les curriculums en santé [5].

Les cursus dits « flexnériens » (du nom de celui qui les a inspirés au début $\mathrm{du} \mathrm{xx}^{\mathrm{e}}$ siècle, Abraham Flexner) partent d'un postulat selon lequel il faut d'abord avoir beaucoup de connaissances biomédicales avant d'apprendre à soigner. Cette organisation curriculaire, qui prédomine notamment en France, se traduit par le fait que les études de santé commencent par plusieurs semestres ou années de formation théorique ciblée sur des disciplines telles que la biologie, l'anatomie, l'embryologie, la biophysique, l'histologie, etc.

Dans les années 1960, des enseignants canadiens ont proposé une alternative afin de répondre à l'un des problèmes majeurs associés aux cursus flexnériens : arrivés dans les années cliniques, les étudiants ont oublié la plupart des 
connaissances biomédicales apprises au début de leur formation. Dans les cursus de type « APP » (pour « apprentissage par problèmes »), qui ont essentiellement été déployés en Amérique et en Europe du Nord, toute connaissance est apprise dans le cadre de l'analyse d'un problème de santé. Concrètement, les étudiants sont dès le début de leur formation médicale soumis à des cas cliniques dont la compréhension (puis la résolution) nécessite qu'ils apprennent et mettent en lien des savoirs issus de plusieurs disciplines. Il s'agit là d'une forme très avancée de contextualisation des apprentissages, c'est-à-dire du fait d'apprendre en étant confronté à des situations qui ressemblent à celles rencontrées en milieu professionnel.

Le déploiement d'un cursus APP implique la mobilisation de ressources considérables, peu envisageables dans le contexte français actuel. En revanche et notamment dans le cadre des réformes du premier et du deuxième cycle des études de médecine, il sera indispensable que les enseignants s'inspirent de certains principes de l'APP, y compris - et surtout - lors du recours au traditionnel et indétrônable (parce qu'économiquement très rentable) cours magistral [5].

\section{Évaluer en contexte authentique}

L'évolution des pratiques d'enseignement devra évidemment s'accompagner d'une modification des modalités d'évaluation, afin de respecter le principe d'alignement pédagogique (qui renvoie à la cohérence entre ce que l'on veut que les étudiants apprennent, la façon d'enseigner et la façon d'évaluer) [5]. Dans l'approche par compétences, on privilégie ainsi l'authenticité des situations d'évaluation, puisque l'objectif n'est plus d'évaluer les connaissances, mais la capacité des étudiants à les utiliser pour résoudre des problèmes en situation professionnelle. Il s'agit de faire en sorte que ces situations ressemblent le plus possible aux situations de soins auxquelles seront confrontés les futurs médecins [5]. Comme il est inenvisageable d'évaluer les étudiants dans toutes les situations qu'ils rencontreront, il est d'usage de regrouper ces situations en familles de situations, en fonction de caractéristiques communes [3].

La simulation offre un niveau d'authenticité potentiellement élevé (on parle parfois de milieu « semi-authentique ») et l'opportunité de standardiser les situations d'évaluation (ce qui est impossible en milieu clinique, qualifié « d'authentique »), afin de répondre aux exigences docimologiques de la certification [5].

\section{Défis de l'évaluation des compétences}

Le travail réalisé dans ce contexte par Philippon et al. [6] offre un éclairage très intéressant sur les enjeux liés à l'éva- luation, auxquels la spécialité de médecine d'urgence est confrontée dans le cadre des réformes du deuxième et du troisième cycle. Il rend compte d'une démarche scientifique d'identification des familles de situation en médecine d'urgence et des critères et indicateurs permettant d'évaluer les étudiants de façon valide et fidèle. L'étude, publiée dans ce numéro des Annales françaises de médecine d'urgence [6], a également pour mérite de révéler les difficultés auxquelles pourraient être exposés les enseignants en médecine d'urgence lors du déploiement des nouvelles modalités d'évaluation imposées par les réformes. Il s'agit, tel que le relèvent les auteurs, de s'approprier les concepts issus de l'approche par compétences (dont celui de « famille de situation », très éloigné des principes qui sous-tendent les pratiques actuelles de formation et d'évaluation), de pallier les limites de l'évaluation en contexte semi-authentique (où les mannequins dits « de haute-fidélité » sont en réalité souvent des freins à l'authenticité, et donc, à l'évaluation des compétences), et de déterminer la forme que devrait prendre le score et ses différents éléments constitutifs [6].

Les auteurs soulignent en outre un point très important, car indissociable de l'évaluation dans l'approche par compétences : il n'est pas possible de se contenter d'observer qu'un étudiant est performant pour le certifier. Autrement dit, cocher des cases en regardant un étudiant agir en simulation est très largement insuffisant pour en conclure qu'il est (ou non) compétent. Comme le font remarquer Philippon et al., le débriefing, systématiquement utilisé pour former en simulation, devrait également être obligatoire pour évaluer en simulation. L'objectif est de s'assurer que le raisonnement qui sous-tend le comportement d'un étudiant est pertinent et que celui-ci n'agit pas par mimétisme, en reproduisant quelque chose qu'il aurait vu faire par un soignant, un enseignant ou un pair, sans comprendre.

\section{Changer la façon de penser la formation et I'évaluation}

Les résultats du travail réalisé par Philippon et al. et la méthode même choisie par les auteurs devraient être largement partagés afin d'alimenter la réflexion actuellement menée dans les différentes disciplines par rapport aux réformes du troisième et du deuxième cycle. Cette dernière prévoit notamment le recours aux examens cliniques objectifs et structurés (ECOS). Les ECOS sont un outil de simulation utilisé massivement et depuis très longtemps dans le monde pour certifier les étudiants en médecine. Leur déploiement dans le cadre d'une approche par compétences est associé à des enjeux que l'article de Philippon et al. permet très clairement d'appréhender.

Plus globalement, le succès (ou l'échec) de ces réformes dépendra de la capacité de leurs promoteurs à modifier en 
profondeur les croyances et les représentations qu'ont souvent les enseignants en médecine sur ce qu'est « un bon cours » ou « une bonne évaluation ». Il s'agira donc de changer la façon de penser tout autant que la façon de faire.

Liens d'intérêts : les auteurs déclarent ne pas avoir de liens d'intérêts.

\section{Références}

1. Tardif J (2006) L'évaluation des compétences : documenter le parcours de développement. Chenelière-Éducation, Montréal
2. Le Boterf G (1995) De la compétence, essai sur un attracteur étrange. Éditions d'organisations, Paris

3. Jouquan J, Parent F (2015) Comment élaborer et analyser un référentiel de compétences en santé ? De Boeck Supérieur, Louvainla-Neuve

4. Pelaccia T (2016) Comment [mieux] former et évaluer les étudiants en médecine et en sciences de la santé ? De Boeck Supérieur, Louvain-la-Neuve

5. Pelaccia T, Tardif J, Triby E, et al (2011). Comment les médecins raisonnent-ils pour poser des diagnostics et prendre des décisions thérapeutiques? Les enjeux en médecine d'urgence. Ann Fr Med Urgence 1:77-84

6. Philippon AL, Hausfater P, Triby E, Freund Y (2019) Développement d'un outil d'évaluation des compétences des étudiants en médecine d'urgence : une étude nationale par la méthode Delphi. Ann Fr Med Urgence 9:354-61 